\title{
Nano-displacement measurements using spatially multimode squeezed light
}

\author{
N. Treps $\dagger+\delta$, N. Grosse $\ddagger$, W. P. Bowen $\ddagger$, M. T. L. Hsu $\ddagger$, A. \\ Maître†, C. Fabre†, H.-A. Bachor $\ddagger$ and P. K. Lam $\ddagger$ \\ $\ddagger$ Australian Centre for Quantum-Atom Optics, Department of Physics, Australian \\ National University, Canberra ACT 0200, Australia. \\ † Laboratoire Kastler Brossel, Université Pierre et Marie Curie, case 74, 75252 Paris \\ cedex 05, France
}

\begin{abstract}
.
We demonstrate the possibility of surpassing the quantum noise limit for simultaneous multi-axis spatial displacement measurements that have zero mean values. The requisite resources for these measurements are squeezed light beams with exotic transverse mode profiles. We show that, in principle, lossless combination of these modes can be achieved using the non-degenerate Gouy phase shift of optical resonators. When the combined squeezed beams are measured with quadrant detectors, we experimentally demonstrate a simultaneous reduction in the transverse $x$ - and $y$ displacement fluctuations of $2.2 \mathrm{~dB}$ and $3.1 \mathrm{~dB}$ below the quantum noise limit.

PACS numbers: 42.50.Dv, 42.30.-d, 42.50.Lc
\end{abstract}

Submitted to: J. Opt. B: Quantum Semiclass. Opt.

$\S$ To whom correspondence should be addressed (nicolas.treps@spectro.jussieu.fr) 


\section{Introduction}

Optical images are one of the most important means used to convey information. Quantum fluctuations are inevitably present in the measurement performed on each of the pixels used to record the image. This results in a deterioration in the quality of the information that can be extracted from it. Quantum fluctuations depend on the characteristics of the light used in the measurement, and specifically designed nonclassical states of light must be used to reduce the quantum noise, extending the limits of information read-out from these images. The study of such quantum fluctuations and correlation in images, and of their applications, is now a topic of growing attention from the quantum optics community [1, 2, 3, 4].

The implementation of a general scheme to reduce quantum noise depends largely on the specifics of the information to be extracted from the beam. For instance, in order to improve the optical resolution (i.e. reduce the size of the minimum detail distinguishable in the image), one requires the use of squeezed states of light in a large number of specific transverse modes [11, 5, 6] or N-photon correlated states [7]. These states of light are typically difficult to produce. However, the problem is greatly simplified when one possesses a priori knowledge about the image to be extracted. For example, a postiori information processing performed on the intensity values measured by each pixel of the detector can be implemented. This technique is widely used when measuring the motion of a microscopic transparent particle [8, macro-molecule [9, 10] and atomic scale surface structures 19. Such measurements can yield sensitivity in the nanometer regime, beyond the wavelength of the light used.

In this paper, we will focus on the specific problem of light beam positioning. The average transverse intensity of the beam is assumed to be constant, but its position and orientation in the transverse plane are unknown. Such measurements are widely used in many practical applications, especially in nanotechnology, where accuracy and reproducibility of positioning at atomic scales are required. If coherent light is used, the spatial randomness of photons introduces quantum fluctuations in the position measurement. The sensitivity of positioning is then at the quantum noise limit [19]. It was proposed theoretically [12] and later demonstrated experimentally (in one [13] and two 14 dimensions) that this limit could be circumvented by using light that has spatial ordering of the photons. The motivation of this paper is to present an overview of optical beam positioning, clearly identify the sources of noise, and then propose ways to improve on these measurements. This will enable us to demonstrate the optimisation of the measurement strategy. We finally present a detailed account of the experiment outlined in Ref. [14, and demonstrate that simultaneous below quantum noise measurements of the beam centre for two different spatial axes are possible. 


\section{Theory}

\subsection{Signal and Noise in Displacement Measurements}

In many applications, such as atomic force microscopes or photonic force microscopes [17, 18, beam positioning is measured using quadrant detectors. The sum and difference photocurrents between the quadrants give information on the relative position of the beam. This measurement delivers photocurrents whose mean value is the positioning signal, and fluctuations around the mean is noise which limit the accuracy of positioning.

The theoretical treatment of quantum noise arising from 1D position measurements using split detector was first examined by Fabre et al. [12]. They showed that the quantum noise could be reduced using a squeezed vacuum field in a mode with a $\pi$ phase shift between the halves impinging on each side of the split detector. Fields with such characteristic phase shifts have henceforth been termed flipped modes. In this article, we provide another perspective on that result, demonstrating that the quantum noise can be reduced simultaneously for several orthogonal spatial measurements performed on the beam.

We use the standard description of the positive frequency part of the electric field operator [4], whose transverse dependence is decomposed over a complete basis of orthogonal modes $\left\{u_{i}\right\}$, within the paraxial approximation

$$
\hat{E}^{+}(\vec{\rho}, z, t)=i \sqrt{\frac{\hbar \omega}{2 \varepsilon_{0}}}\left[\sum_{i} \hat{a}_{i}(z, t) u_{i}(z, \vec{\rho})\right] e^{-i \omega(t-z / c)}
$$

where $z$ is along the propagation direction, $\vec{\rho}$ is the two-dimensional transverse coordinate, $\hat{a}_{i}$ is the annihilation operator of a photon in mode $u_{i}$ and the operators satisfy the commutation relation

$$
\left[\hat{a}_{i}, \hat{a}_{j}^{\dagger}\right]=\delta_{i j}
$$

We then consider the slowly varying envelope $\mathcal{E}(\vec{\rho})$ of the field incident on a plane of longitudinal position $z$ (omitted in the equations), which can be expressed as

$$
\hat{\mathcal{E}}^{+}(\vec{\rho})=i \sqrt{\frac{\hbar \omega}{2 \varepsilon_{0}}} \sum_{i} \hat{a}_{i} u_{i}(\vec{\rho})
$$

The photon number operator at a position $\vec{\rho}$ of the transverse plane (proportional to $\left.\left(\hat{\mathcal{E}}^{+}\right)^{\dagger} \hat{\mathcal{E}}^{+}\right)$is given by

$$
\hat{\mathcal{N}}(\vec{\rho})=\sum_{i, j} \hat{a}_{i}^{\dagger} \hat{a}_{j} u_{i}^{*}(\vec{\rho}) u_{j}(\vec{\rho}) .
$$

The general study of measurements performed with several detectors on the transverse plane will be the subject of a future publication. Here, we present a specific approach applicable when the difference is taken between the intensities on two spatial areas of the beam. To be more specific we will assume that the measurement consists of the difference between the left and right sides of the beam. This restriction does not affect the generality of our results, one may simply change the integration domain to 
adapt them to any detector shape. For a split detector, the photon number difference is given by

$$
\hat{N}_{-}=\int_{x<0} \hat{\mathcal{N}}(\vec{\rho}) d^{2} \rho-\int_{x>0} \hat{\mathcal{N}}(\vec{\rho}) d^{2} \rho=\sum_{i, j} I\left(u_{i}, u_{j}\right) \hat{a}_{i}^{\dagger} \hat{a}_{j}
$$

where $x$ is the horizontal co-ordinate and $I\left(u_{i}, u_{j}\right)$ is defined by

$$
I\left(u_{i}, u_{j}\right)=\int_{x<0} u_{i}^{*}(\vec{\rho}) u_{j}(\vec{\rho}) d^{2} \rho-\int_{x>0} u_{i}^{*}(\vec{\rho}) u_{j}(\vec{\rho}) d^{2} \rho
$$

This equation shows that the pertinent quantities in the calculation of noise are the overlap integrals between the different transverse modes. In order to conveniently evaluate these integrals, we will choose an adapted transverse basis as proposed in Ref. [12, 13]. The first mode in our basis is chosen to match the transverse electric field profile of the beam under interrogation, which we denote by $u_{0}(\vec{\rho})=\mathcal{E}(\vec{\rho}) /\|\mathcal{E}\|$ (This may not necessarily be a $\mathrm{TEM}_{00}$ mode). Since the mean value of the electric field is completely contained in $u_{0}$, the subsequent modes in our basis therefore have no coherent amplitude and only contain fluctuation terms. We now choose the mode $u_{1}$ to be

$$
\begin{array}{lll}
u_{1}(\vec{\rho})=-u_{0}(\vec{\rho}) & \text { if } & x<0 \\
u_{1}(\vec{\rho})=u_{0}(\vec{\rho}) & \text { if } & x>0
\end{array}
$$

where $u_{1}$ is referred to as the flipped mode of $u_{0}$. As the origin of the transverse co-ordinate $x=0$ is chosen to be the centre of the detector, the flipped mode is orthogonal to $u_{0}$ only in the case when the beam is centred on the detector, i.e. when the intensities on the two areas considered are equal. As we would like to measure very small displacements to this configuration, we will consider here that the beam is perfectly centred. For any given spatial basis, Eq. (7) can be used to generate a corresponding flipped mode basis. In this basis, we have seen that the mean values of the annihilation and creation operators are zero for all the modes except $u_{0}$. Hence only overlap integrals that contain the first mode, $I\left(u_{i}, u_{0}\right)$, contribute to Eq. (5) . Using Eq. (17), overlap integrals are given by

$$
I\left(u_{i}, u_{0}\right)=\int u_{i}^{*}(\vec{\rho}) u_{1}(\vec{\rho}) d^{2} \rho=\delta_{i, 1}
$$

which means that only $I\left(u_{1}, u_{0}\right)$ is non-zero. The noise contribution from all other modes are zero. Using the linearised approximation, the noise in the differential measurement can then be written as [12, 15]

$$
\begin{aligned}
\left\langle\delta \hat{N}_{-}^{2}\right\rangle & =N_{\text {tot }}\left\langle\left(\delta \hat{a}_{1}^{\dagger}+\delta \hat{a}_{1}\right)^{2}\right\rangle \\
& =N_{\text {tot }}\left\langle\left(\delta \hat{X}_{1}^{+}\right)^{2}\right\rangle
\end{aligned}
$$

Hence, noise in this measurement arises only from a given quadrature (namely $\hat{X}_{1}^{+}=$ $\hat{a}_{1}+\hat{a}_{1}^{\dagger}$ ) of the flipped mode. It is important to note that this calculation was performed for a beam centred at the detector. However it is still valid for displacements small compared with the beam waist. 
For several simultaneous position measurements performed, for instance with a quadrant detector, one can apply the same method to show that noise arises from the corresponding flipped mode associated with the differential measurement. It is then possible to write an equation similar to Eq. (9) for each differential measurement with its corresponding flipped mode. The orthogonality ensures that the reduction of measurement noise associated with each mode can be independently performed.

\subsection{Engineering Spatially Multimode Squeezed Light}

For a given measurement, the precedent analysis gives the requirement for improving the sensitivity. Indeed, one has to determine the flipped modes involved in the measurement. These modes have then to be produced in a squeezed vacuum in order to reduce the noise in the displacement measurement. As they are orthogonal it is possible, at least theoretically, to spatially overlap them without any losses, and then to spatially overlap the resulting beam with the beam we would like to measure. Furthermore, the fact that the flipped modes are orthogonal implies that it is necessary to use as many squeezed vacuum sources as the number of measurements we would like to improve simultaneously.

Several strategies can be adopted to produce low intensity squeezed beams whose transverse profile is a flipped mode. Shaping the phase of an optical beam can be easily done with a phase mask. However, these devices can be lossy. As we need only to perform $\pi$ phase shifts several schemes have been proposed - one involving a half wave plate, the other a Sagnac interferometer [16. However, other devices such as the ones used in adaptive optics can be implemented [22]. Assuming that the flipped mode can be easily produced without losses (as was the case in our experiment), a sensible strategy would be to produce a squeezed beam with an optical parametric amplifier, and then reshape its phase profile. However, for more complicated schemes, another possibility could be the initial production of a coherent state in the desired transverse mode followed by squeezing using for instance a multimode optical parametric amplifier 21, 4 .

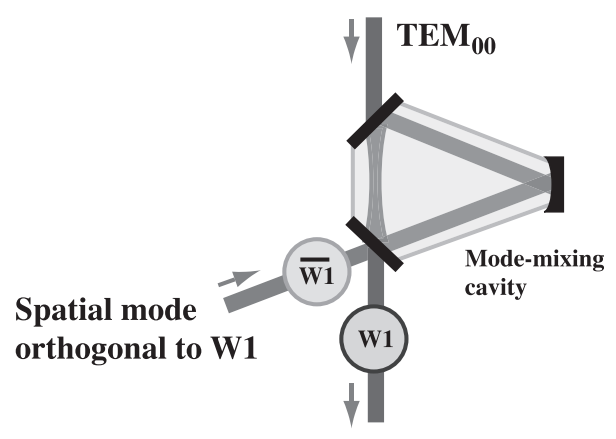

Figure 1. Experimental scheme to spatially overlapping two beams with orthogonal spatial modes using an impedance matched cavity.

Techniques to effectively spatially overlap the modes are, in general, dependent on 
the specific properties of the modes involved, and can be quite complicated. However, so long as the modes involved can be easily produced with wave-plates, any two modes can be overlapped with high efficiency using the mode dependence of an optical cavity. One example of this would be to design a ring optical cavity close to transverse degeneracy, but far enough away to have a good separation of the modes. The cavity can then be tuned to be resonant for the $\mathrm{TEM}_{00}$ mode and far off resonance for all the other modes.

With reference to Fig. 1] the mixing of two transverse modes can be performed in the following way. We begin with one field already in its desired mode-shape, and the other in a $\mathrm{TEM}_{00}$ mode transformable to its desired mode shape using the waveplate $W 1$. Given that we only perform phase shifts, there exists a wave-plate $\bar{W} 1$ that performs the reverse operation to that performed by $W 1$. Our $\mathrm{TEM}_{00}$ mode is transmitted through an impedance matched cavity, and the wave-plate $W 1$ is placed after the cavity to produce the desired mode. The second mode is then reflected off the empty port of the cavity output mirror. However, as the mode will also go through wave-plate $W 1$, it is thus necessary to transmit the mode through wave-plate $\bar{W} 1$ prior to the cavity. The resulting effect of the wave-plate combination $\bar{W} 1 W 1=I$ ( $I$ is the identity operation) on the second mode, is a preservation of its initial mode-shape. In this way, the transmitted and reflected modes are, in principle, perfectly spatially overlapped, with losses avoided by an appropriate design for the optical cavity.

Other schemes to spatially overlap the beams can be proposed for specific cases. For instance, it often appears that one needs to mix an odd mode with an even mode. In this case, a Mach-Zehnder like interferometer can be utilised, as used in the single photon community [20]. In that article, the Mach-Zender was used as a transverse-mode beam splitter. It is possible to use it the other way around, as a lossless transverse-mode beam mixer, demonstrated in our scheme.

\section{Optimised Displacement Measurement}

\subsection{Beam Imaging}

One of the main applications of precise laser beam positioning is the measurement of physical effects coupled to the beam displacement. For instance, in atomic force microscopy, a laser beam is reflected off a cantilever and its position is recorded by a quadrant detector. One can then examine the optimal methods to extract information from the physical system using that technique. The previous calculation demonstrated that, using a coherent beam, the quantum noise in the measurement depends only on the intensity of the beam. For a given intensity, and a given detector shape, a pertinent question is: how can we obtain the largest signal, and hence the largest signal to noise ratio?

If we consider only $\mathrm{TEM}_{00}$ Gaussian beams, we have shown in a previous publication 12 that, in the case of a coherent state impinging on an infinitely broad split detector, 
the displacement corresponding to a signal to noise ratio of one is given by

$$
d=\sqrt{\frac{\pi}{8}} \frac{w_{0}}{\sqrt{N}}
$$

where $N$ is the total number of photons and $w_{0}$ the beam waist. Given this formula, for a fixed laser intensity, it appears that the relevant quantity is the displacement divided by the beam waist. Using lenses to increase the beam size, proportionally increases the relative displacement, and therefore does not change their ratio. The consequence of this is that the precision of the measurement will remain the same. The best configuration to measure a displacement induced by a physical system is to apply the displacement to the smallest beam possible to maximise this ratio [19]. The maximum focussing is the wavelength divided by the numerical aperture of the optical system [24]. The smallest measurable displacement induced by a physical system coupled to a coherent laser beam is therefore given by

$$
d_{S Q L}=\sqrt{\frac{\pi}{8}} \frac{\lambda}{2 N A \sqrt{N}}
$$

where $\lambda$ is the wavelength and $N A$ the numerical aperture of the focussing lens.

Let us now consider a detector whose two halves have a finite size $D$ with a gap $\delta$ in between, used to measure a beam displacement. Changing the beam size with lenses does not affect the precision of the measurement only in the case of an infinite detector, however here the losses induced both by the gap and the detector size are important. The best signal to noise ratio occurs when minimum loss is experienced by the beam. In this case it it therefore important to optimise the beam waist size to minimise the total losses induced by the gap and the detector edges.

\subsection{Data Acquisition}

In practice, at low frequencies the quantum noise limit for displacement measurements is overwhelmed by classical noise arising from acoustic or seismic vibration sources coupling to the system. Therefore measurements of the amplitude of periodic displacements (i.e. displacement modulation) were chosen to be made at high frequencies $\nu_{\text {mod }}>1 \mathrm{MHz}$ where such noise sources were negligible. An electronic spectrum analyser (ESA) can be chosen to extract the spectral power density of the displacement signal. This corresponds to a measurement of the oscillation amplitude rather than an absolute displacement.

The refined task is then to measure the square of the amplitude of the periodic displacement within a small frequency range of oscillations as a function of time. An ESA has two parameters that require specification (RBW and VBW). The resolution bandwidth ( $\mathrm{RBW}$ in units of $\mathrm{kHz}$ ) determines the small range of frequencies included in the measurement around the central frequency $\Omega_{\bmod }$. The band-passed signal is then demodulated at $\Omega_{\text {mod }}$ and its amplitude detected. The measurement time $T$ is given by the inverse of the RBW setting. $N$ photons are collected in this time, setting the level of noise power measured on the ESA. In the absence of displacement modulations, 
this level corresponds to the quantum noise, and as such is used as a reference and calibration for further displacement modulation measurements.

The response of an ESA is non-trivial for the combination of Gaussian noise and a signal [23]. For a displacement modulation signal $d_{\text {mod }}$ and noise $d_{\text {noise }}$ of the same order $\left(d_{\text {mod }} \approx d_{\text {noise }}\right)$, the response of the ESA in units of displacement squared may be approximated as $\left(d_{\text {total }}^{2}=d_{\text {mod }}^{2}+d_{\text {noise }}^{2}\right)$. The calibration from spectral noise power to displacement is determined as follows - for a laser beam of power $P$, frequency $\nu$, waist size $\omega_{0}$, and an ESA setting of RBW, the number of photons collected per measurement is given by $N=P / h \nu \mathrm{RBW}$, which determines $d_{\mathrm{SQL}}$ from Eq. (11).

In order to set the calibration constant, a trace from the ESA was obtained where $d_{\text {mod }}$ was kept to zero, hence giving the noise power level corresponding only to the quantum noise limit contribution. Another ESA trace of $d_{\text {total }}$ where $d_{\text {mod }}$ was nonzero was then obtained. The smallest identifiable displacement $\left(d_{\min }\right)$ can be defined in terms of the statistical confidence that a displacement modulation has been observed. One complication of using an ESA to identify displacement modulations is that the video bandwidth (VBW in units of $\mathrm{Hz}$ ) operation of an ESA sets the averaging length over several $\Omega_{\text {mod }}$ oscillation cycles of $d_{\text {total }}$, and thus changes the time response of our displacement measurement. However, for practical applications where we require a time resolution equal to the one set by the resolution bandwidth, we simply choose the video bandwidth equal to the resolution bandwidth.

In summary, an efficient quantum noise limited displacement modulation measurement can be achieved by carefully focusing the beam on the sample and detector, and choosing appropriate spectrum analyser settings. After optimisation of these parameters, there remain only two ways to improve the quality of the measurement - increasing the mean power or using spatial squeezing. It is the latter solution that we implement and describe in the following section.

\section{2-D Spatial Squeezing - Experimental Scheme and Results}

\subsection{Quadrant Detection}

Our quadrant detector, composed of a $2 \times 2$ array of separate square detectors of side $0.5 \mathrm{~mm}$ and adjacent separation of $25 \mu \mathrm{m}$ (Epitaxx ETX 505Q), allows us to perform 4 independent measurements (see Fig. 2)

$$
\begin{aligned}
& I_{a}=I_{1}+I_{2}+I_{3}+I_{4} \\
& I_{b}=\left(I_{1}+I_{4}\right)-\left(I_{2}+I_{3}\right) \\
& I_{c}=\left(I_{1}+I_{2}\right)-\left(I_{3}+I_{4}\right) \\
& I_{d}=\left(I_{1}+I_{3}\right)-\left(I_{2}+I_{4}\right)
\end{aligned}
$$

The first measurement, $I_{a}$ corresponds to the sum of the four quadrants and gives the total intensity. $I_{b}$ and $I_{c}$ correspond respectively to horizontal and vertical positioning. Finally, $I_{d}$, which is the difference between the two diagonal, can be interpreted, to 
the first order, as an orientation measurement. Indeed, if the beam is for instance elliptical, $I_{d}$ is zero only if the axis of the ellipse is parallel to the quadrants. To each of these measurements corresponds a flipped mode defined by Eq. (7). In the case of a $\mathrm{TEM}_{00}$ input beam, the three flipped modes are orthogonal, and it is then possible to improve all 3 differential measurements simultaneously. We will show here how we could improve any two of them simultaneously, in a configuration easily scalable to the three measurements. Experimentally, the challenges in this process are to efficiently

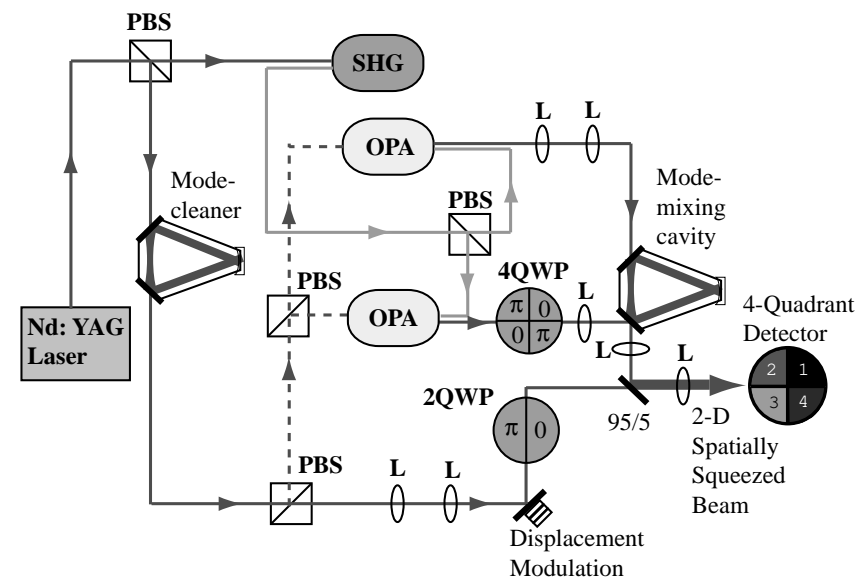

Figure 2. Schematic of experimental setup for the production of a 2-dimensional spatially squeezed beam and its detection scheme. PBS: polarising beam-splitter, SHG: second-harmonic generator, OPA: optical parametric amplifier, 4QWP: 4-quadrant wave-plate, 2QWP: 2-quadrant wave-plate, L: imaging lens, 95/5: Beam-splitter with $95 \%$ reflection and $5 \%$ transmission.

produce the correct spatial modes, to generate a pair of squeezed fields, and to combine these fields with the coherent horizontally flipped mode with maximum efficiency. Our experimental setup, which overcomes these challenges, is shown in Fig. 2.

\subsection{Generation of Squeezed Light}

In our experiment a $1.5 \mathrm{~W}$ field at $1064 \mathrm{~nm}$ was generated by a non-planar ring-oscillator Nd:YAG laser. This field was split into two roughly equal parts. One part was used to pump a second harmonic generator (SHG), and the other was ultimately used to seed a pair of optical parametric amplifiers (OPAs) each generating a squeezed field, and to provide the coherent horizontally flipped field. The SHG was constructed from a hemi-lithic $\mathrm{MgO}: \mathrm{LiNbO}_{3}$ crystal with curved surface AR coated at $1064 \mathrm{~nm}$ and $532 \mathrm{~nm}$, and an input/output coupling mirror with $92 \%$ reflectivity for $1064 \mathrm{~nm}$ light and $\sim 5 \%$ reflectivity for $532 \mathrm{~nm}$ light. An electro-optical modulation was applied to the $\mathrm{MgO}: \mathrm{LiNbO}_{3}$ crystal, resulting in a phase modulation on the intra-cavity field. An error signal for the length of the cavity was extracted by detecting the light transmitted through the cavity and mixing down at the modulation frequency. The SHG was non-critically phase matched by holding the temperature of the $\mathrm{MgO}: \mathrm{LiNbO}_{3}$ crystal 
constant at $\sim 107^{\circ} \mathrm{C}$. At this temperature, a conversion efficiency of $\sim 55 \%$ was achieved. This resulted in $350 \mathrm{~mW}$ of optical power at $532 \mathrm{~nm}$, which was used to pump our OPAs.

The second $1064 \mathrm{~nm}$ beam was passed through a mode cleaning cavity. This cavity had a line-width of $2 \mathrm{MHz}$ and so acted to filter out noise on the laser above that frequency. Consequently, the output field was quantum noise limited at frequencies above $\sim 6 \mathrm{MHz}$. It was then used to seed both of our OPAs and to provide the horizontally flipped coherent field. The OPAs were of identical design to the SHG, but with higher output coupler reflectivity at $1064 \mathrm{~nm}$ (96\%). As with the SHG, the length of each OPA cavity was controlled using an error signal generated through an intra-cavity phase modulation. Each OPA either amplified or de-amplified its seed field, depending on the relative phase between the pump and seed. The SHG phase modulation resulted in a phase modulation on the OPA pump fields, and consequently a modulation of the OPA amplification. For each OPA, an error signal could be extracted from this modulation, locking the OPA to de-amplification. In this regime the OPAs each produced a $150 \mu \mathrm{W}$ amplitude squeezed field. These squeezed fields were observed to be $4 \mathrm{~dB}$ and $3 \mathrm{~dB}$ below the quantum noise limit, respectively.

\subsection{Manipulation of Transverse Mode-Shapes}

At this stage, we had available all of the resources required to generate a $2 \mathrm{D}$ spatially squeezed beam, all that remained was to appropriately modify each beam's transverse mode-shape, to generate a spatial modulation, and to spatially overlap the beams. In our experiment, for practical reasons, instead of using a $\mathrm{TEM}_{00}$ laser we chose to have the mean field in a horizontally flipped mode $\mathrm{TEM}_{\mathrm{f} 00}$. Hence, the flipped mode for horizontal positioning is simply a $\mathrm{TEM}_{\mathrm{f} 00}$ mode and the one for vertical positioning is a doubly flipped mode $\mathrm{TEM}_{\mathrm{f} 0 \mathrm{f} 0}$. To summarise, we required the bright coherent field to have a horizontally phase flipped mode-shape $\mathrm{TEM}_{\mathrm{f} 00}$, and the dim squeezed fields to have $\mathrm{TEM}_{00}$, and both horizontally and vertically flipped $\mathrm{TEM}_{\mathrm{fof} 0}$ mode-shapes, respectively. The required mode-shape modification could be achieved by phase flipping some quadrants of each field. This phase flipping was achieved using birefringent optical half wave-plates which were cut and assembled at appropriate $\pi / 2$ angles (CSIRO Lindfield Australia). A 2-quadrant wave-plate was used on the local oscillator beam so that its mode-shape was horizontally phase flipped, while a 4-quadrant wave-plate was used on one squeezed beam so that its mode-shape was both horizontally and vertically flipped. The other squeezed beam required no modification and was maintained as a $\mathrm{TEM}_{00}$ Gaussian mode.

In order to gauge the quality of the phase flipped wave-plates fabricated, we interfered the flipped modes with either another flipped mode or the $\mathrm{TEM}_{00}$ mode (see Fig. 3). This was performed using a Mach-Zehnder interferometer setup where one arm of the interferometer had a TEM $_{\text {f00 }}$ wave-plate and the other arm had either a TEM $_{\text {fofo }}$ wave-plate or no wave-plate. The input modes at the interferometer were 
of equal optical power and by scanning the relative phase between the beams in the two arms, interference fringes on each quadrant of the 4-quadrant detector (labelled 1, 2, 3 and 4 here as defined in Fig. (3) were observed. We quantified the quality of the mode-matching via the interference fringe visibility

$$
V=\frac{I_{\max }-I_{\min }}{I_{\max }+I_{\min }}
$$

where $V=1$ indicates perfect mode-matching and $V=0$ indicates no mode-matching.

When a $\mathrm{TEM}_{00}$ mode was interfered with a horizontally flipped mode (as shown in Fig. 目 A) the interference observed on the half-detector defined by quadrants 2 and 3 was $\pi$ out of phase with that on the half-detector defined by quadrants 1 and 4 , as expected since the phase of the $\mathrm{TEM}_{\mathrm{f} 00}$ field was flipped by $\pi$ between the halves. The measured visibility of the interference was $V=0.90$ and the phase mismatch between the constructive and destructive interference was $7^{\circ}$. As expected, when a $\mathrm{TEM}_{\mathrm{fof0}}$ mode was interfered with the $\mathrm{TEM}_{\mathrm{fo0}}$ mode (as shown in Fig. [3 B) the interference observed on the half-detector defined by quadrants 1 and 2 was $\pi$ out of phase with that on the half-detector defined by quadrants 3 and 4 . In this case, the visibility of the interference was $V=0.88$ and phase mismatch was $5^{\circ}$. The effective efficiency of our mode-shape modification procedure is given by $V^{2}$, so that the efficiency for both wave-plate designs was approximately $80 \%$.
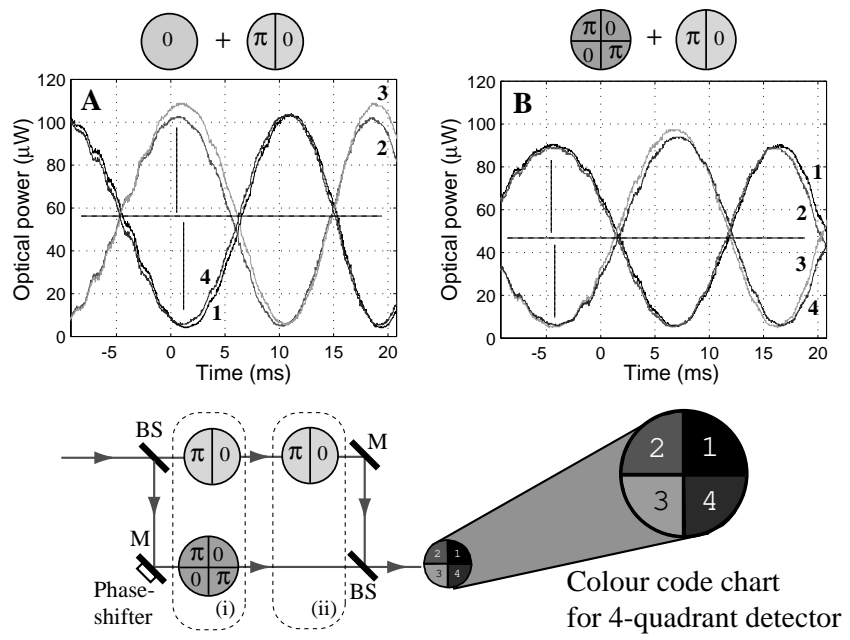

Figure 3. Plot A: interference of $\mathrm{TEM}_{00}$ mode with $\mathrm{TEM}_{\mathrm{f} 00}$ mode. Plot B: interference of $\mathrm{TEM}_{\mathrm{fof0}}$ mode with $\mathrm{TEM}_{\mathrm{f} 00}$ mode. The vertical lines indicate the times when constructive and destructive interference occurs. The Mach-Zehnder setup is shown where wave-plate combination (i) interrogates the interference between the $\mathrm{TEM}_{\mathrm{fof0}}$ and $\mathrm{TEM}_{\mathrm{fo0}}$ modes and (ii) interrogates the interference between the $\mathrm{TEM}_{00}$ and $\mathrm{TEM}_{\mathrm{f} 00}$ modes. The numbers $(1,2,3,4)$ on the plots indicate the measurement results corresponding to the quadrants of the detector. BS: beam-splitter, M: mirror 


\subsection{Overlapping the Spatial Modes}

To obtain a single 2D spatially squeezed beam, the three modes produced above were spatially overlapped. Since squeezing is highly sensitive to inefficiencies, it was critical that the efficiency of the process overlapping the squeezed modes was high. An element that accurately distinguished between different spatial modes was required to achieve this high efficiency. In our case we chose a specially designed optical cavity in a ring configuration as this element. Cavities constructed using spherical mirrors decompose incident light fields into the $\mathrm{TEM}_{\mathrm{mn}}$ modes. The Gouy phase shift between the $\mathrm{TEM}_{\mathrm{mn}}$ modes typically ensures that for a given cavity length some $\mathrm{TEM}_{\mathrm{mn}}$ modes will be resonant, and others entirely non-resonant. In our case, we designed the cavity to be impedance matched, and have relatively low finesse $(\sim 31)$. The input and output couplers had $95 \%$ reflectivity and the third mirror was HR coated. In this regime losses in the cavity were small compared to the input and output coupling, and resonant incident modes were transmitted with high efficiency. With a cavity length of $200 \mathrm{~mm}$ controlled such that the $\mathrm{TEM}_{00}$ mode was resonant, our $\mathrm{TEM}_{00}$ squeezed beam was transmitted with $>95 \%$ efficiency and a line-width of $25 \mathrm{MHz}$. We were interested in squeezing at sideband frequencies of $\sim 5 \mathrm{MHz}$. This is well below the line-width of the cavity, ensuring efficient transmission of our squeezing.

Let us now consider the $\mathrm{TEM}_{\mathrm{fof} 0}$ squeezed mode interacting with the cavity. Since the cavity was in a ring configuration, reflection of this mode from the cavity output coupling mirror with appropriate imaging lenses achieved the desired spatial overlap with the transmitted $\mathrm{TEM}_{00}$ mode (see Fig. 1). Of course, for this reflection to be efficient the $\mathrm{TEM}_{\mathrm{fof} 0}$ had to be far way from the cavity resonance. The resonance condition of the $\mathrm{TEM}_{\mathrm{f} 0 \mathrm{fo}}$ mode for the ring-cavity can be examined by first expanding it in the $\mathrm{TEM}_{\mathrm{mn}}$ basis

$$
\begin{aligned}
& \mathrm{TEM}_{\mathrm{fOf0}}=\sum_{i=0}^{\infty} \sum_{j=0}^{\infty} c_{m n} \mathrm{TEM}_{\mathrm{mn}} \\
& c_{m n}=\frac{(-1)^{i+j}(2 i) !(2 j) !}{\pi(i !)(j !) 2^{i+j-1} \sqrt{(2 i+1) !(2 j+1) !}}
\end{aligned}
$$

where $m=2 i+1$ and $n=2 j+1$. Notice that there is no contribution to the $\mathrm{TEM}_{\mathrm{fof} 0}$ mode from the $\mathrm{TEM}_{00}$ mode. Thus, these two modes are orthogonal, and there is no fundamental limitation on the efficiency with which they can be spatially overlapped. A number of cavity designs exist for which this overlap, in principle, approaches $100 \%$ efficiency. The crucial factor is that when the $\mathrm{TEM}_{00}$ mode is on resonance, all higher order $\mathrm{TEM}_{\mathrm{mn}}$ mode with significantly large occupation $\left|c_{m n}\right|^{2}$ are far off resonance. In the limit of high cavity finesse this condition is naturally satisfied.

In our case, however, a relatively low cavity finesse was chosen to ensure the efficient transmission of squeezing at $5 \mathrm{MHz}$ on the $\mathrm{TEM}_{00}$ squeezed mode. In this case, a careful analysis of the resonance condition of each of the significant higher order $\mathrm{TEM}_{\mathrm{mn}}$ modes is required. For each mode, this resonance condition is dictated by the Gouy phase shift experienced by the modes, which can be optimised for a given cavity length through 
an appropriate choice of the radii of curvature of the cavity mirrors. For our cavity the input/output coupling mirrors were chosen to be flat and the third mirror to have a radius of curvature of $250 \mathrm{~mm}$. With the cavity length controlled so that the $\mathrm{TEM}_{00}$ mode was resonant, all higher order modes with $n+m<18$ were efficiently reflected. The contribution to our TEM $_{\text {fofo }}$ mode from TEM modes with $n+m>18$ was insignificant with $\left|c_{m n}\right|^{2}<0.0003$ in all cases. For our cavity configuration, therefore, the $\mathrm{TEM}_{\mathrm{fof} 0}$ mode was efficiently reflected. We directly observed an efficiency $>94 \%$ for this process.

Fig. 4 shows a comparison of the predicted and experimentally observed cavity reflection for a $\mathrm{TEM}_{\mathrm{fof} 0}$ mode as a function of the cavity resonance condition. The cavity resonance condition was scanned by ramping the cavity length using a piezoelectric actuator (PZT) attached to the curved cavity mirror. When a TEM $_{\mathrm{mn}}$ mode was resonant, it was almost entirely transmitted, and the reflected power from the cavity dropped in consequence. The magnitude of the intensity drop was directly proportional to $\left|c_{m n}\right|^{2}$. Fig. 4, therefore, illustrates the significance of each of the lowerorder $\mathrm{TEM}_{\mathrm{mn}}$ modes constituting the $\mathrm{TEM}_{\mathrm{fof} 0}$ mode. The highest contribution came from the $\mathrm{TEM}_{11}$ mode, and the significance of the modes dropped rapidly as $m+n$ increased. A comparison of Fig. 4 A and B shows a good agreement between the theory and experiment. The experimental peaks were slightly broader than the prediction which can be attributed to the intra-cavity loss.

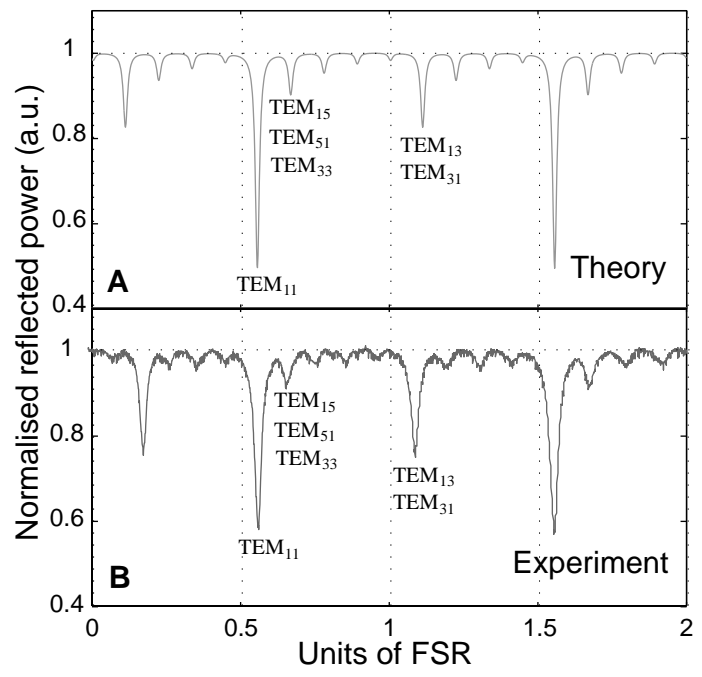

Figure 4. Theoretical (A) and experimental (B) intensity plots for $\mathrm{TEM}_{\mathrm{fof0}}$ reflection from the ring-cavity. For the theoretical reflection plot, the reflected intensity was computed for the first $m=n=29 \mathrm{TEM}_{\mathrm{mn}}$ modes with cavity parameters equal to the cavity used in the experiment.

Transmitting the $\mathrm{TEM}_{00}$ squeezed beam through our cavity, and simultaneously reflecting the $\mathrm{TEM}_{\mathrm{fof} 0}$ squeezed beam from the cavity mirror spatially overlapped the two fields. All that remained to generate a 2D spatially squeezed beam was to spatially overlap this combined beam with our bright coherent TEM $_{\mathrm{f} 00}$ mode. Since the second 
order statistical moments of coherent fields are insensitive to attenuation, this overlap could be achieved by utilising a $95 / 5$ beam splitter. The combined squeezed field, and the coherent flipped field were imaged onto the two input ports of the beam splitter. The combined squeezed field was reflected with $95 \%$ efficiency, and the flipped coherent field was transmitted with $5 \%$ efficiency. the resulting output field was 2D spatially squeezed.

\subsection{D Spatial Squeezing Measurements}

The level of squeezing exhibited by our spatially squeezed beam was examined by imaging the mode onto a quadrant detector. The horizontal beam displacement fluctuation was obtained by taking the difference between the photocurrents originating from the left and right halves of the quadrant detector, and the vertical beam displacement fluctuation was obtained from the difference of the photocurrents from the top and bottom halves of the detector. These photocurrents were then analysed in a spectrum analyser which performed power spectral density measurements. Without squeezing, these measurements were limited to the quantum noise level as shown by the black trace in Fig. 5. Introducing the squeezed fields, we observed simultaneous squeezing of both the horizontal and vertical displacement fluctuations as shown by the colour (grey) traces in Fig. [5. The optimum simultaneous squeezing for the vertical and horizontal displacement fluctuations were $2.0 \pm 0.1 \mathrm{~dB}$ and $3.05 \pm 0.1 \mathrm{~dB}$, respectively. This corresponded to a reduction in the displacement fluctuations of the vertical and horizontal axes by $37 \%$ and $50 \%$, respectively, with respect to the quantum noise level.

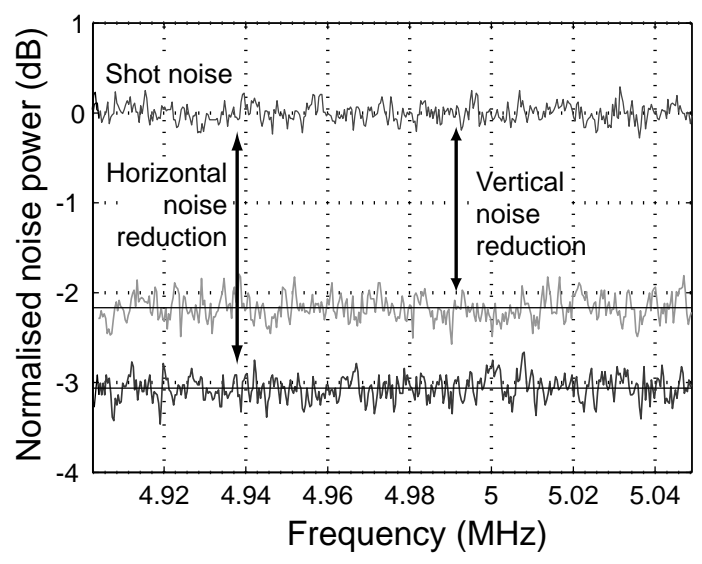

Figure 5. The plot at $0 \mathrm{~dB}$ (black) is the quantum noise level. The plots at $2.2 \mathrm{~dB}$ (green) and $-3.1 \mathrm{~dB}$ (red) are the respective noise levels for the vertical and horizontal axes of the 2-dimensional spatially squeezed beam. Spectrum analyser settings: $150 \mathrm{kHz}$ span at $4.976 \mathrm{MHz} . \mathrm{RBW}=100 \mathrm{kHz}, \mathrm{VBW}=100 \mathrm{~Hz}$, averaged over 10 traces. 


\subsection{Implementation and Measurement of a Displacement Modulation}

With a reduction in the noise levels of both the horizontal and vertical axes to below the quantum noise limit, we now examine the effects of an externally applied displacement modulation on the spatially squeezed beam. Spatial squeezing should improve the resolution with which this modulation can be analysed.

The external displacement modulation was applied to the $\mathrm{TEM}_{\mathrm{f} 00}$ local oscillator beam by reflecting the beam from a $45^{\circ}$ angled mirror mounted on a PZT modulated at $4.976 \mathrm{MHz}$. The PZT consisted of a stack of piezo-electric crystals. When a voltage was applied across the PZT, the PZT not only contracted/expanded in the principal axis but also tilted at an angle away from the principal axis. The resulting transverse modulation from the PZT was a diagonal displacement modulation, with horizontal and vertical components.

In order to demonstrate an improvement in the resolution measurement of our 2-dimensional spatially squeezed beam, we compared the results of a displacement modulation measurement between a coherent beam and our spatially squeezed beam. The results are shown in Fig. [6. The squeezing levels obtained for the horizontal and vertical axes were $2.84 \mathrm{~dB}$ and $1.80 \mathrm{~dB}$, respectively. The broad peak corresponded to the measured displacement modulation signal and we measured our signal to noise ratio (SNR) from the maximum of the signal peak to the noise floor. For the quantum noise limited coherent beam (curve (i)), the measured SNR for the horizontal and vertical axes were 2.8 and 1.4. For the 2-dimensional spatially squeezed beam (curve (ii)), the measured SNR for the horizontal and vertical axes were 5.2 and 1.9. The improvement in the measurement of the displacement modulation signal obtained from the spatially squeezed beam over the coherent beam is given by the ratio of $\mathrm{SNR}_{\mathrm{sqz}}^{\mathrm{H}(\mathrm{V})} / \mathrm{SNR}_{\text {coherent }}^{\mathrm{H}(\mathrm{V})}$. The respective ratios obtained for the horizontal and vertical axes were 1.9 and 1.4.

We also examined the effects of ramping the displacement modulation amplitude over time. To determine the displacement modulation amplitude at an analysis frequency $\Omega$, the photocurrents from the quadrant detector were analysed using a spectrum analyser. The spectrum analyser demodulated the modulation signal and then performed a measurement of the power spectral density. The displayed information was the sum of the squares of the quantum noise without modulation $d_{\text {noise }}(\Omega)$ and the small transverse beam position modulation $d_{\text {mod }}(\Omega)$ - i.e. $d_{\text {noise }}(\Omega)^{2}+d_{\text {mod }}(\Omega)^{2}$ (see Fig. 7A). Curve (i) is the result of the measurement performed using a coherent state and thus is the best classical measurement. Curve (ii) was obtained by performing the same measurement, but with a spatially squeezed beam instead.

The data obtained in Fig. [7A was then normalised to the respective noise levels for

the coherent and the spatially squeezed beams, shown in Fig. 7B. The vertical axis is the difference between the measured signal, and noise without displacement. For the spatially squeezed beam the average of the signal trace crosses the threshold of confidence for a smaller modulation amplitude than for the coherent beam. The corresponding oscillation amplitude for the spatially squeezed and coherent beams were found to be 


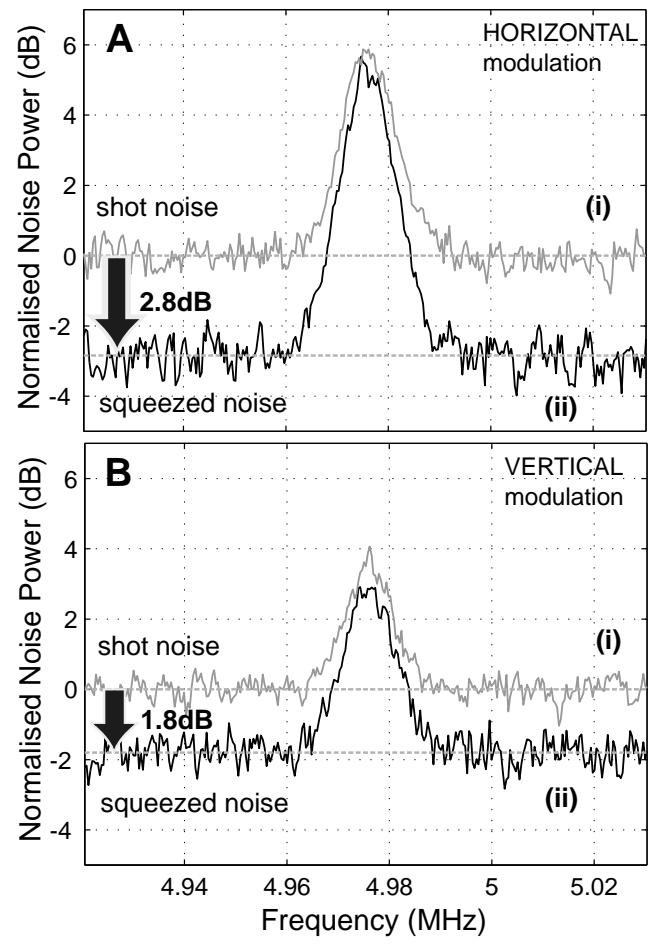

Figure 6. Curves (i) in plots A and B (yellow) are the measured displacement modulation for a coherent beam in the horizontal and vertical axes, respectively. Curves (ii) in plots A and B (black) are the measured displacement modulation for a 2-dimensional spatially squeezed beam in the horizontal and vertical axes, respectively. Spectrum analyser settings : $150 \mathrm{kHz}$ span at $4.976 \mathrm{MHz}, \mathrm{RBW}=10 \mathrm{kHz}$, VBW $=100 \mathrm{~Hz}$, averaged over 20 traces.

$1.6 \AA$ and $2.3 \AA$ at the $99 \%$ confidence level. Thus the improvement for the spatially squeezed beam was a factor of 1.5 over the quantum noise limited coherent beam. Since both traces increased linearly with the modulation amplitude, this improvement factor was independent of the choice of confidence levels.

For the sake of brevity, we have only included the results for the horizontal axis. It should be realised that an equivalent improvement for two simultaneous measurements performed in two orthogonal quadratures on the spatially squeezed beam has been achieved.

\subsection{Simultaneous 2D Displacement Measurement}

An intuitive visualisation of our 2D spatial squeezed beam was obtained by analysing the vertical and horizontal normalised amplitude quadratures on a correlation diagram. To do this, simultaneous measurements of the horizontal and vertical beam displacements were required. We achieved these measurements using a pair of simultaneously triggered spectrum analysers with identical settings. The results for the two axes could then be plotted against each other in conventional correlation diagrams. Fig. 8 shows the resulting correlation diagrams for a quantum noise limited beam (Fig. 8 A) and our 

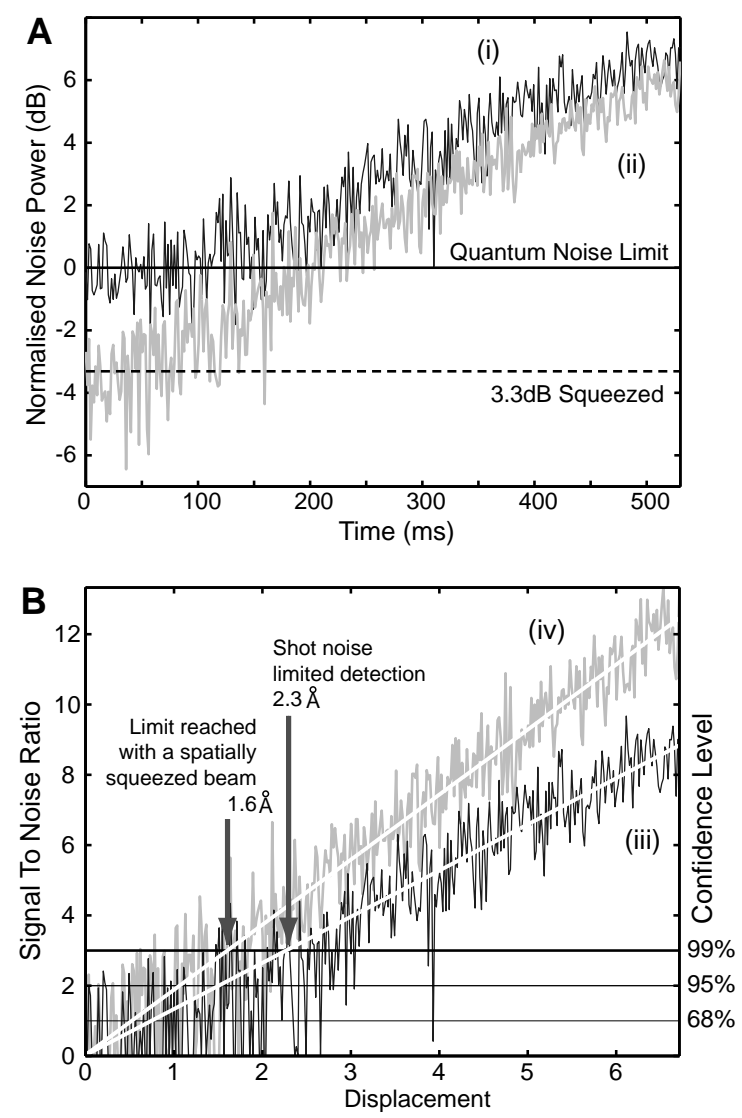

Figure 7. Plot A shows the results of a horizontal displacement measurement signal ramped up in time, with (i) obtained from a coherent beam and (ii) from a spatially squeezed beam. In plot B, the signal-to-noise improvement (left vertical axis) is plotted against the inferred displacement. Traces (iii) and (iv) show the results from data traces (i) and (ii), respectively. Spectrum analyser settings: $\mathrm{RBW}=\mathrm{VBW}=1 \mathrm{kHz}$, averaged over 20 traces each with detection time $\triangle t=1 \mathrm{~ms}$ per data point.

spatially squeezed beam (Fig. 8 B). Each point in these diagrams can be interpreted as an instantaneous 2D measurement of the fluctuations of the displacement modulation. The standard deviation as a function of modulation angle could be calculated for each correlation diagram, and is displayed by the ellipses in the figure. We can directly see that the average displacement modulation fluctuation for all angles is smaller with our spatially squeezed beam than with a coherent beam. Specifically, for the horizontal and vertical axes, the average fluctuation is reduced by a factor of 0.78 and 0.75 , respectively. The square of these values give the fluctuation variances of $2.2 \mathrm{~dB}$ and $2.5 \mathrm{~dB}$ for the horizontal and vertical axes, respectively. The ellipses in Fig. 8 also allow an analysis of any correlation present between the horizontal and vertical displacement fluctuations. Since in both cases the major and minor ellipse axes correspond well to the horizontal and vertical displacement axes, it is clear that no cross-axis correlation exists. 


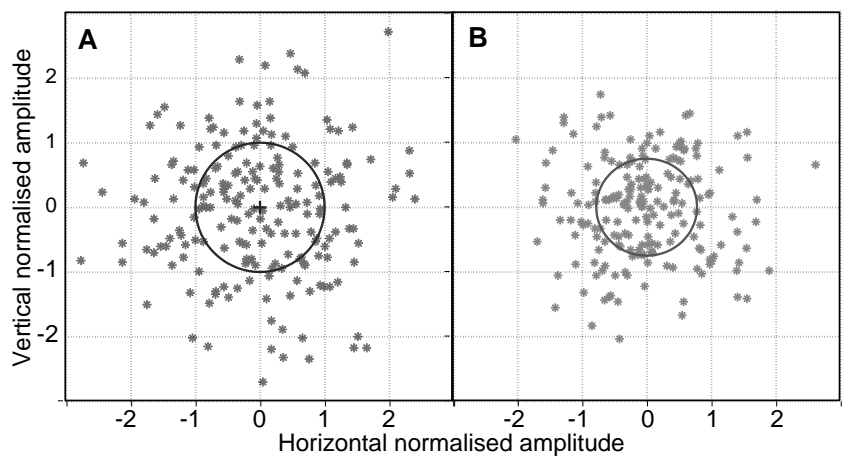

Figure 8. The quantum noise data points (blue) are shown in plot A and the squeezing points (red) are shown in plot B. The quantum noise mean value is indicated by the blue + sign at the centre of plot $\mathrm{A}$. The blue and red circles indicate the standard deviations for the quantum noise and squeezing data, respectively. Re-scaling was implemented such that the quantum noise standard deviation equalled one. The two spectrum analysers had settings of $0 \mathrm{~Hz}$ span at $4.976 \mathrm{MHz}, \mathrm{RBW}=\mathrm{VBW}=1 \mathrm{kHz}$, averaged over 6 traces.

\subsection{Extension to Diagonal Axis Measurement}

As discussed in the theory section, simultaneous enhancement of any two orthogonal spatial properties of a beam is possible given access to sufficient squeezing resources, and the ability to choose and combine appropriate spatial modes. In this section, we demonstrate that principle by simultaneously generating horizontal and diagonal spatial squeezing, contrasting the horizontal/vertical spatial squeezing discussed throughout the majority of this paper. Our bright coherent beam and $\mathrm{TEM}_{00}$ squeezed beam were maintained in their original modes, and the 4-quadrant wave-plate in the $\mathrm{TEM}_{\mathrm{fof} 0}$ squeezed beam was replaced by a 2-quadrant wave-plate, rotated by $90^{\circ}$ relative to the wave-plate for the coherent beam. This transformed the TEM $\mathrm{T}_{\text {fofo }}$ squeezed beam to a vertically flipped $\mathrm{TEM}_{0 \mathrm{fo}}$ mode. So that, in this case, the beams involved were a bright coherent horizontally flipped $\mathrm{TEM}_{\mathrm{f} 00}$ beam, a vertically flipped $\mathrm{TEM}_{0 \mathrm{fo}}$ squeezed beam and a $\mathrm{TEM}_{00}$ squeezed beam. To measure the diagonal axis modulation displacement, the photocurrents from diagonal detector quadrants were summed, and the difference of the two resulting photon currents were analysed in a spectrum analyser. The resulting horizontal and diagonal displacement modulation spectra are shown in Fig. 9. As can be seen from the figure, the horizontal and diagonal axes were simultaneously squeezed by $2.2 \mathrm{~dB}$ and $1.8 \mathrm{~dB}$, respectively. This corresponded to displacement fluctuation reductions with respect to quantum noise of $40 \%$ and $34 \%$, for the horizontal and diagonal axes respectively.

\subsection{Efficient One Dimensional Spatial Squeezing}

In our original one dimensional (1D) spatial squeezing work reported in Ref. [13, the spatially squeezed beam was produced by overlapping a squeezed $\mathrm{TEM}_{00}$ beam and a 


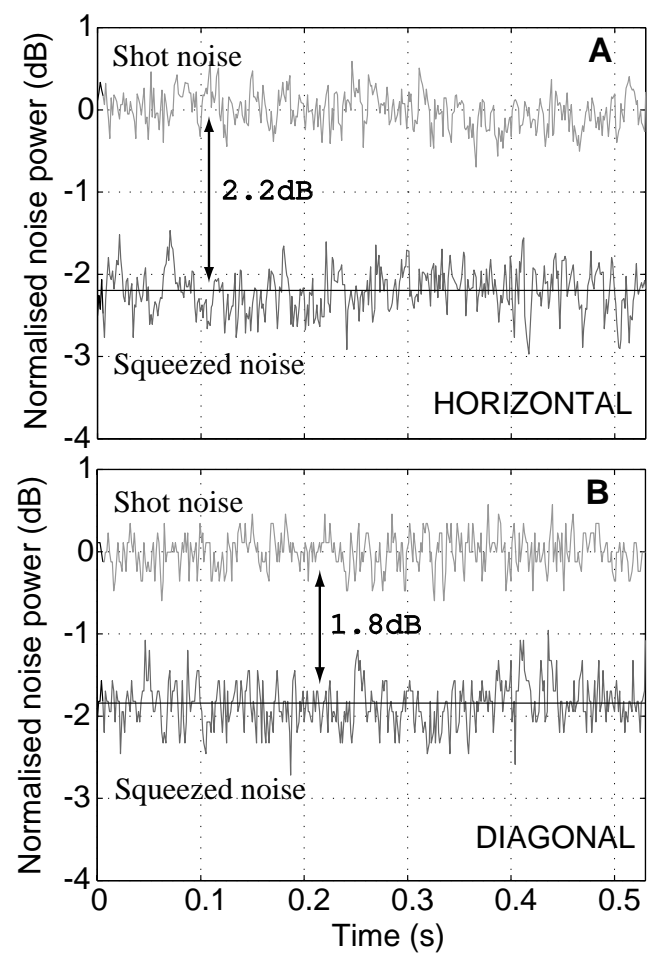

Figure 9. Horizontal (A) and diagonal (B) axes squeezing spectra. Spectrum analyser settings: $0 \mathrm{~Hz}$ span at $4.976 \mathrm{MHz}, \mathrm{RBW}=100 \mathrm{kHz}, \mathrm{VBW}=100 \mathrm{~Hz}$, no averaging.

bright coherent $\mathrm{TEM}_{\mathrm{f} 00}$ beam using a $95 / 5$ beamsplitter. The choice of $95 \%$ reflectivity was motivated by the requirement to minimise loss in the $\mathrm{TEM}_{00}$ squeezed beam, while ensuring a significant fraction of the coherent beam was transmitted. However, this resulted in an attenuation of the displacement signal encoded on the coherent beam, so that the achievable signal-to-noise ratio was lower than would be possible if the spatial overlap was achieved efficiently. We repeated that original experiment, using our cavity to efficiently spatially overlap the two beams. This resulted in a 1D spatially squeezed beam with minimal losses for both the squeezed and coherent beams. The measured squeezing was $2.5 \mathrm{~dB}$, which was also an improvement on the value of $2.34 \mathrm{~dB}$ reported in Ref. [13].

\section{Conclusion}

In this paper we have investigated, both theoretically and experimentally, the optimum displacement measurements that can be performed using a detector array. We demonstrated that, splitting the array into two arbitrary areas with equal optical power on each, enhancement of the difference measurement between the halves could be achieved using a $\pi$ phase-flipped squeezed mode. The location of the $\pi$ phase-flip was dictated by the detector areas under investigation. We then showed that an arbitrary number of such measurements could be enhanced simultaneously using multiple squeezed 
beams, so long as the squeezed beams had orthogonal spatial modes.

In our experiment, we were interested in enhancing measurements performed using an array detector with only four pixels, or in other words, a quadrant detector. Such measurements can be interpreted as displacement measurements, and have many applications. Our aim was to use squeezing to achieve simultaneous squeezing of multiple displacement measurements. One of the primary experimental challenges was to develop methods to spatially overlap two beams with orthogonal spatial modes efficiently. We achieved this overlap with approximately $95 \%$ efficiency using a specially designed optical resonator. We used this resonator to spatially overlap a pair of quadrature squeezed beams with specifically configured mode-shapes. Combining the resulting beam with a bright coherent beam, also with a specific mode-shape, produced a 2D spatially squeezed beam. This was demonstrated by simultaneous below shot noise fluctuations in both horizontal and vertical displacement measurements when the beam was imaged onto our quadrant detector. We introduced a spatial modulation to the beam and demonstrated that, using spatial squeezing, a modulation amplitude of $1.6 \AA$ could be detected with $99 \%$ confidence, a coherent beam, on the other hand, could only detect modulations with amplitude greater than $2.3 \AA$. To illustrate the generality of out results, we also demonstrated that horizontal and diagonal displacement measurements could be simultaneously enhanced.

\section{Acknowledgment}

We acknowledge financial support from the Australian Research Council Centre of

Excellence Program and the European network QUANTIM, contract IST-2000-26019. We thank Roman Schnabel, Ben Buchler and Ulrik Andersen for assistance and fruitful discussions. The Laboratoire Kastler-Brossel of the Ecole Normale Superieure and University Pierré et Marie Curie are associated via CNRS.

[1] L.A. Lugiato, A. Gatti and E. Brambilla, J. Opt. B, 4, S176 (2002).

[2] A. Joobeur, B.E.A. Saleh, T.S. Larchuck and M.C. Teich, Phys. Rev. A 53, 5349 (1996).

[3] H. Lee, P. Kok and J.P. Dowling, quant-ph/0306113

[4] M.I. Kolobov, Rev. Mod. Phys., 71, 1539 (1999).

[5] M.I. Kolobov and I.V. Sokolov, Phys. Lett. A, 140, 101-103 (1989).

[6] L.A. Lugiato and P. Grangier, J. Opt. Soc. Am. B, 14, 225 (1997).

[7] J.J. Perina, B.E.A. Saleh and M.C. Teich, Phys. Rev. A, 57, 3972 (1998); A.N. Boto, P. Kok, D.S. Abrams, S.L. Braunstein, C.P. Williams and J.P. Dowling, Phys. Rev. Lett. 85, 2733 (2000).

[8] W. Denk and W.W. Webb, Appl. Opt., 29, 2382 (1990).

[9] S. Kamimura, Appl. Opt., 26, 3425 (1987)

[10] J. Jelles, B.J. Schnapp and M.P. Scheetz, Nature, 331, 450 (1988).

[11] M.I. Kolobov and C. Fabre, Phys. Rev. Lett., 85, 3789 (2000).

[12] C. Fabre, J.B. Fouet and A. Maître, Optics Letters, 25, 76-78 (1999).

[13] N. Treps, U. Andersen, B. Buchler, P.K. Lam, A. Maître, H.-A. Bachor and C. Fabre, Phys. Rev. Lett., 88, 203601 (2002).

[14] N. Treps, N. Grosse, W.P. Bowen, C. Fabre, H.-A. Bachor and P.K. Lam, Science, 301, 940 (2003).

[15] N. Treps, PhD thesis, Laboratoire Kastler Brossel, Université Pierre et Marie Curie (2001).

[16] V. Delaubert, D.A. Shaddock, P.K. Lam, B.C. Buchler, H.-A. Bachor and D.E. McClelland, J. Opt. A, 4, 393 (2002). 
[17] T.J. Senden, Current Opinion In Colloid 63 Interface Science, 6, 95-101 (2001).

[18] A. Rohrbach, Proceedings of the European Topical Meeting on Advanced Imaging Techniques, Delft, NL, (2003).

[19] C.A.J. Putman, B.G.De Grooth, N.F. Van Hulst and J. Greve, J. Appl. Phys., 72, 6 (1992).

[20] H. Sasada and M. Okamoto, Phys. Rev. A 68, 012323 (2003)

[21] M.I. Kolobov and I.V. Sokolov, Sov. Phys. JETP, 69, 1097 (1989).

[22] J. Courtial and M.J. Padgett, Opt. Commun., 159, 13 (1999).

[23] D. A. Hill and D. P. Haworth, IEEE Transactions on Instrumentations and Measurements, 39, $432(1990)$.

[24] A.E. Siegman, Lasers, University Science Books (1986). 